\title{
One Indication for an Extravascular Cardiac Resynchronization Therapy Defibrillator: Lessons from a Combination Therapy Case with Epicardial Cardiac Resynchronization Therapy and a Subcutaneous Implantable Cardioverter Defibrillator
}

\author{
Natsuko Ishii ${ }^{1}$, Kenzaburo Nakajima ${ }^{1,2}$, Takashi Kakuta ${ }^{3}$, Takashi Noda ${ }^{1}$, \\ Tomoyuki Fujita ${ }^{3}$ and Kengo Kusano ${ }^{1,2}$
}

\begin{abstract}
:
Extravascular cardiac resynchronization therapy (CRT) defibrillators (CRT-Ds) are ideal for recurrent blood stream infections. Furthermore, CRT is useful for patients intolerant to right ventricular (RV) pacing. The case was a 65-year-old man with a CRT-D who presented with a blood stream infection. Because he was hemodynamically unstable with temporary RV pacing, an epicardial CRT device was re-implanted concomitantly through a surgical procedure. After the operation, a subcutaneous implantable cardioverter defibrillator (S-ICD) was placed. However, not all pacing is eligible for S-ICD screening. Combination therapy with an epicardial CRT device and S-ICD might be an alternate option for cardiac surgery cases.
\end{abstract}

Key words: S-ICD, CIED infection, epicardial CRT, cross talk

(Intern Med 60: 1877-1880, 2021)

(DOI: 10.2169/internalmedicine.6125-20)

\section{Introduction}

With improvements and increases in the lead extraction experience and instruments, removing leads is more common than before. However, we occasionally experience patients with a reduced left ventricular ejection fraction (LVEF) who do not tolerate right ventricular (RV) pacing while awaiting re-implantation, especially in infected lead extraction cases. To avoid recurrent blood infections with cardiac implantable electronic devices (CIEDs), the surgical implantation of an epicardial cardiac resynchronization therapy (CRT) device in combination with a subcutaneous implantable cardioverter defibrillator (S-ICD), known as a complete "extravascular device", is ideal.

The feasibility of the implantation of a S-ICD and epicardial pacemaker has already been reported (1). However, there are no guidelines regarding which cases are indicated for the combination of an epicardial left ventricular (LV) lead and S-ICD. To our knowledge, this is the first report of limitations associated with epicardial pacing in combination therapy for patients with severe left ventricle dysfunction who are intolerant to RV pacing.

\section{Case Report}

A 65-year-old man with a history of inferior myocardial infarction presented with recurrent Staphylococcus aureus bacteremia. He also was implanted with a CRT defibrillator (CRT-D; Boston Scientific, Massachusetts, USA, RESONATE X4, model G447, RA lead: INGEVITY MRI 7,741, RV lead: RELIANCE 4-FRONT 0692, LV lead: ACUITY X4 Straight 4,672) for the secondary prevention of ventricular fibrillation (VF) and ischemic cardiomyopathy. The device had been implanted via the left subclavian vein for five months. He was transferred to our hospital for further treat-

${ }^{1}$ Department of Cardiovascular internal medicine, National Cerebral and Cardiovascular Center, Japan, ${ }^{2}$ Graduate School of Medical Sciences Kumamoto University, Japan and ${ }^{3}$ Department of Cardiovascular surgery, National Cerebral and Cardiovascular Center, Japan Received: August 19, 2020; Accepted: October 29, 2020; Advance Publication by J-STAGE: February 1, 2021 Correspondence to Dr. Kengo Kusano, kusanokengo@hotmail.com 
A

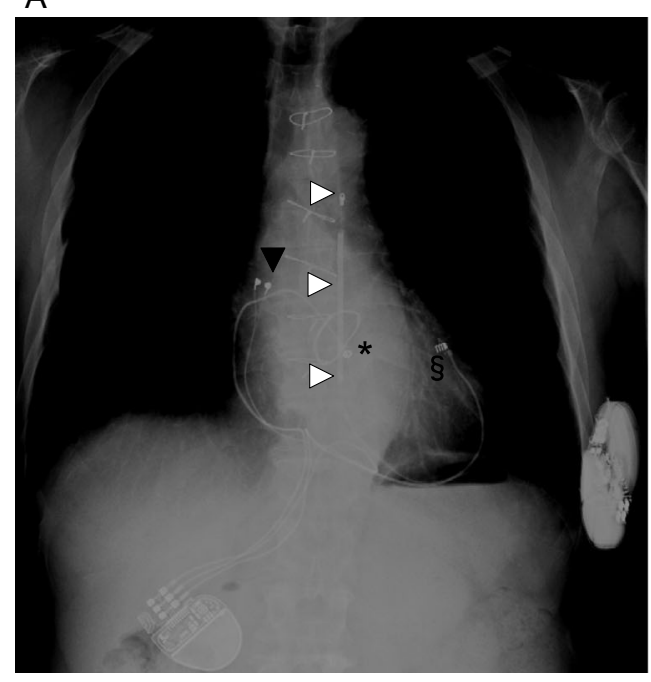

B

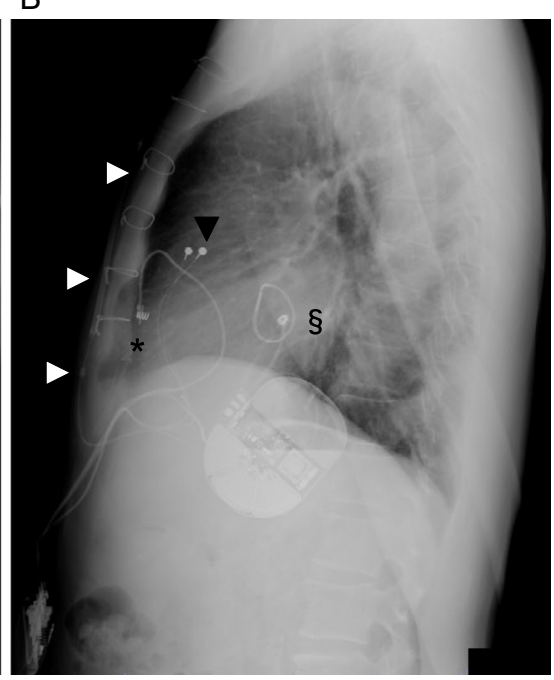

Figure 1. Chest radiographs after the implantation of a subcutaneous implantable cardioverterdefibrillator (S-ICD) and permanent epicardial cardiac resynchronization therapy (CRT) device. The can of the S-ICD is positioned in a subcutaneous pocket on the lower left-lateral thorax. The can of the permanent CRT device is positioned above the abdominal fascia, as far as possible from the $S$ ICD. A: Posterior-anterior chest radiograph post-implantation of the S-ICD and permanent epicardial CRT device. B: Lateral view of the chest radiograph. The white triangle indicates that the electrode was not attached to the sternal wire. Black triangle: RA bipolar lead. *: RV lead. §: LV lead

ment.

His vital signs were stable under an infusion of antibiotics. However, his bacteremia was not controlled because of a device infection. His atrioventricular node dysfunction progressed due to beta blockers, and he was dependent on biventricular pacing. Transthoracic echocardiography revealed that his left ventricle ejection fraction was $30 \%$ and that he had severe functional mitral regurgitation. We performed transesophageal echocardiography twice, finding no evidence of infective endocarditis. A coronary angiogram revealed significant narrowing of both the left and right coronary arteries.

Initially, we attempted to extract the lead transvenously, but a significant reduction in the LV outflow tract-velocity time integral (VTI) suggested worsening heart failure due to the RV pacing after the removal of the infected CIED. In this case, there was no guarantee that venoarterial extracorporeal membrane oxygenation (ECMO) would keep the patient hemodynamically stable without any further infections.

First, the patient completed a six-week course of intravenous cefazolin. While the bacterial blood culture was negative, there was some fluid retention around his device. Therefore, he underwent removal of the infected transvenous CRT-D, coronary bypass graft, and mitral valve plication electively once his bacterial culture was negative.

During the surgery, bipolar permanent epicardial leads (right atrial lead: Medtronic, Minneapolis, USA, Capsure Epi bipolar lead; right ventricle/left ventricle lead: Medtronic, Myodex bipolar lead) were placed on the right atrium, right ventricle, and lateral left ventricle and were connected to a triple-chamber CRT device (Boston Scien- tific; VALITUDE, model U125), and the CRT-P generator was implanted in an abdominal location (Fig. 1). Three epicardial leads were implanted without any problems: right atrial (RA) lead: $1.6 \mathrm{~V} / 0.5 \mathrm{~ms}$, $\mathrm{P}$ wave $4.0 \mathrm{mV}$, and impedance $500 \Omega$; RV lead: $1.5 \mathrm{~V} / 0.5 \mathrm{~ms}, \mathrm{R}$ wave $4.0 \mathrm{mV}$, and impedance $600 \Omega$; and LV lead: $2.7 \mathrm{~V} / 0.5 \mathrm{~ms}$, R wave $3.0 \mathrm{mV}$, and impedance $600 \Omega$. We continued intravenous antibiotics for four weeks postoperatively. An implantable cardioverter defibrillator (ICD) system was necessary for the secondary prevention of VF. However, concerning the high risk of a recurrent infection, an S-ICD was ideal for him.

\section{Procedure}

Screening for epicardial pacing was performed with 54 patterns using an EMBLEM ${ }^{\mathrm{TM}}$ automated screening tool (AST). Only bi-ventricular pacing was eligible (Fig. 2). The S-ICD was implanted using a two-incision technique. Fluoroscopy was used just for marking the left side of the parasternum to avoid attaching the thoracic wire and to determine the pocket location (2). Considering cross talk between the S-ICD and epicardial pacemaker, which has previously been reported (3), we were able to manage the oversensing of the pacing and sensing between the epicardial CRT device and S-ICD. When the pacing output was programmed to $7.5 \mathrm{~V}$, which was the highest output of the epicardial CRT device, the S-ICD was not sensed as a double or triple count.

When inducing VF, the CRT was programmed to VVI at 90 beats/min, all paced at a maximal output of $7.5 \mathrm{~V} / 1.5$ $\mathrm{ms}$, and the CRT ventricular sensing was set at $4 \mathrm{mV}$, which was the lower sensitivity. The tachycardia-sensing lower rate 


\section{BiV pacing RV pacing LV pacing}

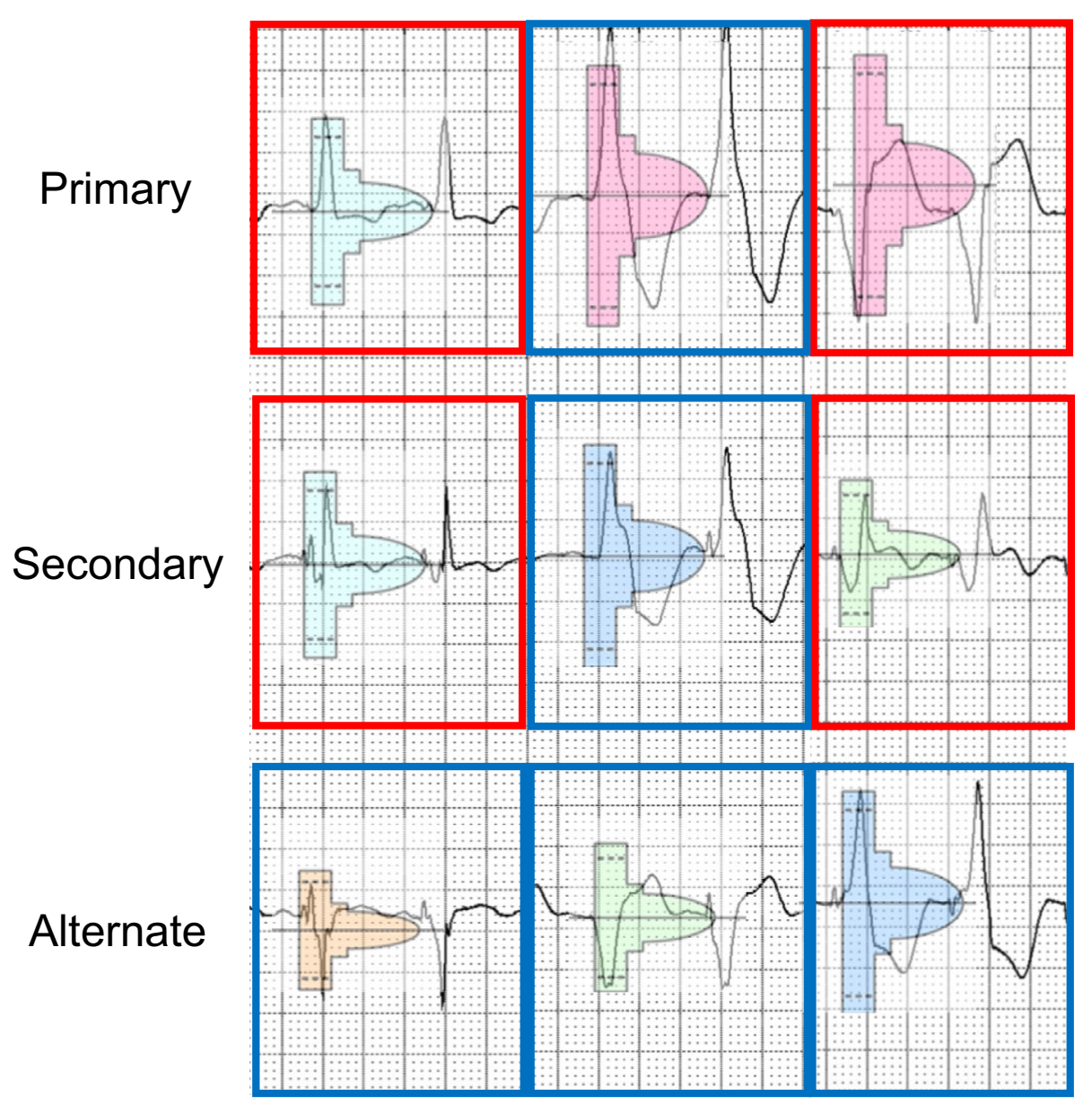

Figure 2. Eligible and ineligible leads for S-ICD lead screening on the left parasternal region for each type of pacing: biventricular (BiV), right ventricular ( $R V)$, and left ventricular $(\mathrm{LV})$. The red rectangles mean eligible, and the blue rectangles mean ineligible.

of the S-ICD was set at 200 beats/min. During the defibrillation threshold (DFT) testing, the S-ICD sensed the VF, did not sense the pacing spike from the CRT device and delivered appropriate shock therapy. After the successful implantation of the S-ICD, the patient was discharged without any heart failure exacerbation or recurrent blood stream infection.

\section{Discussion}

The feasibility of the combination of an S-ICD and epicardial or endocardial pacemaker has been described, and favorable outcomes have already been reported $(1,3,4)$. However, all of the cases in those studies were implanted with only an LV pacing device and S-ICD in the same setting. In this case, we demonstrated for the first time an indication for surgical patients who are hemodynamically unstable with regard to RV pacing.

However, several issues associated with pacing need to be kept in mind when these two devices are adopted in a patient, as follows: a) Bipolar pacing leads must be selected for CRT, as unipolar pacing is contraindicated for S-ICDs, and electromagnetic interference might occur between the CRT device and S-ICD. The safety feature of the automatic conversion from bipolar to unipolar pacing must be turned off.

b) The programmed pacing AV delay needs to be adequately shortened for complete bi-ventricular pacing because it depends on the AV conduction and can differ between intrinsic QRS waves and paced waves. It is impossible to screen all patterns, and in the present case, the complete atrioventricular block made it easy to screen only the biventricular pacing. At the same time, the AV delay should be set in order not to automatically switch to another pacing mode (e.g., Smart Delay or multisite pacing should not be programmed).

c) We have to turn off the measurement of the thoracic impedance in the CRT device in order to prevent cross talk with the S-ICD. This will help prevent the generator from being reset and causing undersensing of VF in response to applicable non-recoverable or repeat fault conditions after delivering a shock. 
d) The upper tracking rate was programmed to half of the shock detecting zone in order to avoid double counting. In this case, the CRT maximum tracking rate was programmed to 120 paces/minute. The S-ICD conditional shock zone was programmed to 200 beats/minute, and the shock zone was programmed to over 250 beats/minute.

e) During DFT testing, the CRT pacing should be programed to the VOO mode at the maximum output, in order to identify the worst-case scenario, which would be undersensing of VF by the S-ICD (3).

Although the major benefit associated with the transvenous re-implantation of a CRT-D is to avoid any interaction between devices and to make future generator exchanges much simpler, combination therapy seems to have a huge advantage. There are no risks of recurrent infections or intolerance to temporary RV pacing during the antimicrobial therapy period. Furthermore, there are no limitations associated with the vascular access or coronary sinus branches for transvenous CRT implantations.

With an automated screening tool before implantation, the RV- and LV-only pacing was out of the appropriate range and the only the bi-ventricular pacing was eligible for $\mathrm{S}$ ICD in this case. It was because with only LV or RV pacing, the QRS width broadened and made it inappropriate for S-ICD screening. Implanting three epicardial leads in the RA, RV, and LV increased the choices with regard to pacing, helping to avoid any inappropriate screening.

\section{Conclusion}

Implantation of an S-ICD and bipolar epicardial CRT-D was feasible and safe. However, there may be limitations associated with S-ICD screening with epicardial CRT pacing. The co-implantation of an S-ICD and epicardial device (extravascular CRT-D) requires care during pacing. It may be adequately safe and feasible in patients with recurrent device infections and heart failure.

The authors state that they have no Conflict of Interest (COI).

\section{References}

1. Steinberg C, Chakrabarti S, Krahn AD, Bashir J. Nothing inside the heart - combining epicardial pacing with the S-ICD. Heart Rhythm Case Reports 1: 419-423, 2015.

2. Angel B, Overcash J, Fischer W, Fontaine JM. Surgical and electrophysiological considerations in the management of a patient with a subcutaneous implantable cardioverter-defibrillator undergoing coronary artery bypass surgery. Heart Rhythm Case Reports $\mathbf{3}$ : 58-62, 2017.

3. Kossidas K, Kalman R, Follis WP, Cooper JM. Managing cross talk between a subcutaneous implantable cardioverter-defibrillator and a dual-chamber unipolar pacemaker system. Heart Rhythm Case Reports 3: 579-583, 2017.

4. Sharma S, Nand NP, Hematpour K, Reddy SK, Salas de Armas IA, Patel MK. Implantation of left atrial-ventricular epicardial pacemaker system and subcutaneous implantable cardioverterdefibrillator in a single setting: the "extravascular" cardiac resynchronization therapy. Heart Rhythm Case Reports 5: 354-358, 2019.

The Internal Medicine is an Open Access journal distributed under the Creative Commons Attribution-NonCommercial-NoDerivatives 4.0 International License. To view the details of this license, please visit (https://creativecommons.org/licenses/ by-nc-nd/4.0/).

(C) 2021 The Japanese Society of Internal Medicine Intern Med 60: 1877-1880, 2021 